\title{
Spin-torque effects in metallic magnetic multilayers in the ballistic regime
}

\author{
P. M. Krstajić* \\ Departement Fysica, Universiteit Antwerpen, Groenenborgerlaan 171, B-2020 Antwerpen, Belgium \\ M. Keller \\ LEM SA, Chemin des Aulx 8, 1228 Plan-les-Ouates, Switzerland \\ F. M. Peeters ${ }^{\dagger}$ \\ Departement Fysica, Universiteit Antwerpen Groenenborgerlaan 171, B-2020 Antwerpen, Belgium \\ (Received 18 December 2007; revised manuscript received 8 April 2008; published 22 May 2008)
}

\begin{abstract}
We derive the current-induced torque in metallic magnetic multilayers in the ballistic regime for the case that one of the ferromagnetic layers is acting as a full polarizer. We generalize previous results to the case when transmission and reflection amplitudes of one of the ferromagnets are different from zero or one and are complex. The obtained expressions are valid for the situation when the interfaces are sufficiently flat and when there are no scatterers in the metallic spacers. Particular attention is paid to multilayers containing a perpendicular polarizer whose magnetization is pointed along the current flow. We present analytical expressions for the spin torque that may serve as a theoretical upper bound for realistic structures.
\end{abstract}

DOI: 10.1103/PhysRevB.77.174428 PACS number(s): 75.70.Cn, 73.40.-c, 75.47.Jn, 75.30.Ds

\section{INTRODUCTION}

Metallic magnetic multilayers are heterostructures made of alternating layers of ferromagnetic and nonmagnetic [normal $(\mathrm{N})$ ] layers that exhibit the giant magnetoresistance effect, ${ }^{1,2}$ i.e., modulation of resistance when the ferromagnetic layers change their relative orientation, usually due to changes in the external magnetic field. ${ }^{3}$ Since its discovery ${ }^{3,4}$ in 1988, there has been an intense research on this phenomenon and it led to applications in sensitive magnetic field sensors such as read heads of contemporary hard disks. However, recently, there has been a growing interest in the inverse effect magnetization reversal induced by spin-polarized current flowing perpendicularly through such structures, as proposed by Berger $^{5}$ and Slonczewski. ${ }^{6}$ Experimentally, current-induced magnetization reversal has been realized in $\mathrm{Co} / \mathrm{Cu} / \mathrm{Co} \quad$ sandwich $\quad$ structures, ${ }^{7,8} \quad \mathrm{Py} / \mathrm{Cu} / \mathrm{Py}$ heterostructures, ${ }^{9}$ and $(\mathrm{Ga}, \mathrm{Mn}) \mathrm{As} / \mathrm{GaAs} /(\mathrm{Ga}, \mathrm{Mn}) \mathrm{As}$ tunnel junctions. ${ }^{10}$

Theoretical approaches proposed in the literature that describe this spin-torque effect depend on the assumptions about the thicknesses of the layers, the quality of the interfaces, and the value of temperature. Especially important is the length and the quality of the normal layer, as to whether its length is smaller or larger than the spin diffusion length of the carriers. Another issue is to resolve whether to take into account ballistic or diffusive transport depending on the number of impurities inside the normal layer. ${ }^{11}$ The approaches found in the literature vary from all-quantum descriptions, ${ }^{5,6}$ diffusive but still phase coherent, ${ }^{11}$ density matrix approach and two-current model, ${ }^{12,13}$ and Green's function method within the current model ${ }^{14}$ to pure driftdiffusion model. ${ }^{15}$ Other references relevant to mention are $a b$ initio approaches ${ }^{16}$ that investigated the problem of the $\mathrm{Cu} / \mathrm{Co}$ interface. ${ }^{17}$

In this paper, we generalize the Slonczewski ${ }^{6}$ result to the case when one of the ferromagnets acts as a full polarizer while the other is characterized by complex transmission and reflection amplitudes for spin majority $\left(t_{u}, r_{u}\right)$ and spin minority $\left(t_{d}, r_{d}\right)$ subbands. An all-quantum description of the spin transfer will be presented, i.e., valid if the interfaces are sufficiently flat and clean and in the absence of scattering centers in the normal metal spacer. Therefore, it is assumed that transport is ballistic but the Fermi length $\lambda_{F}$ is still smaller than the thickness $D_{N}$ of the spacer, and the mean-free path $\left(l_{\mathrm{mf}}\right)$ and spin-flip relaxation length $\left(l_{\mathrm{sf}}\right)$ are still larger than the dimensions of the structure, i.e., $\left\{l_{\mathrm{mf}}, l_{\mathrm{sf}}\right\}>D_{N}>\lambda_{F}$, which implies that phase averaging should be performed inside the spacer.

The paper is organized as follows. In Sec. II, we present the theoretical formalism that enables one to find amplitudes of the electron wave function in the layers of interest and it is assumed that the phase in the nonmagnetic layer may fluctuate. This allows one to estimate the transmission amplitude and the total current, which is independent of the spacer length $D_{N}$ under the above mentioned condition. In Sec. III, we derive analytical formulas for the spin torque acting on the polarizer and discuss its dependence on the phases of the reflection and the transmission amplitudes. In Sec. IV, the model is extended to multilayers containing perpendicular polarizer whose magnetization is parallel to the current flow. In the last section, Sec. V, we summarize our results and present our conclusions.

\section{THEORETIC FORMALISM}

Figure 1 shows the schematics of the spin-valve structure of interest. It consists of two ferromagnetic layers, $\mathrm{FM}_{A}$ and $\mathrm{FM}_{B}$, with the second being a perfect polarizer separated by a nonmagnetic metallic spacer (region 2). The magnetization of the first ferromagnet is tilted over an angle $\theta$ with respect to the second one. It is assumed that the current flows from left to right, and therefore, electrons first impinge on the right ferromagnet, $\mathrm{FM}_{A}$. It is important to emphasize the direction of the moving electrons because the spin torque 


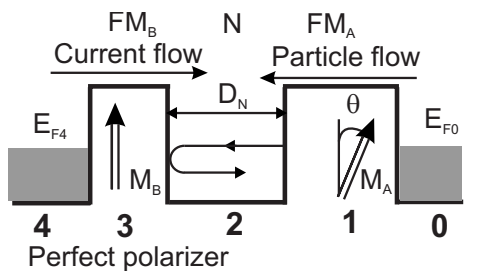

FIG. 1. Schematic diagram of the double barrier structure consisting of two ferromagnetic (FM) layers separated by a nonmagnetic spacer layer. The assumed direction of the current flow is from left to right while electrons flow in the opposite direction. One of the ferromagnetic layers $\left(\mathrm{FM}_{B}\right)$ acts as a full polarizer.

changes its sign ${ }^{11}$ with the interchange of the two ferromagnets. As usual, the applied bias $V_{b}=E_{F 4}-E_{F 0}$ is taken to be significantly smaller than the Fermi energies $E_{F 3,4}$, and the external leads, i.e., regions 0 and 4 , are taken to be identical.

In each $n$th region (0-4), within the flat-band approximation, the wave function is a two-component spinor multiplied by plane waves,

$$
\Psi(x)=\sum_{i=1,2} a_{n, i}\left(\begin{array}{c}
F_{n i \uparrow} \\
F_{n i \downarrow}
\end{array}\right) e^{i x k_{x n, i}},
$$

where the index $i$ runs over the different directions of propagation, i.e., leftward $(L)$ or rightward $(R)$. The quantization axis is chosen to be along the $z$ axis, the same direction as the magnetization of the polarizer, while the propagation of the current is along the $x$ axis (see Fig. 2). This set of equations together with the boundary conditions can be solved by the transfer or scattering matrix approach. However, if one concentrates only on components that propagate in one direction, ${ }^{11}$ here, leftward, it is possible to find a matrix $Q$ that connects the incoming amplitudes $\Psi_{0 L}$ in region 0 and outgoing amplitudes $\Psi_{4 L}$ in region 4,

$$
\left(\begin{array}{c}
\psi_{4 L \uparrow} \\
\psi_{4 L \downarrow}
\end{array}\right)=Q\left(\begin{array}{c}
\psi_{0 L \uparrow} \\
\psi_{0 L \downarrow}
\end{array}\right) .
$$

In the case considered here, the normal metallic spacer introduces only an additional phase, the second ferromagnetic layer $\left(\mathrm{FM}_{B}\right)$ acts as a full polarizer and spin-flip processes are assumed to be negligible. Then, reflection and transmission matrices for $\mathrm{FM}_{A}$ are

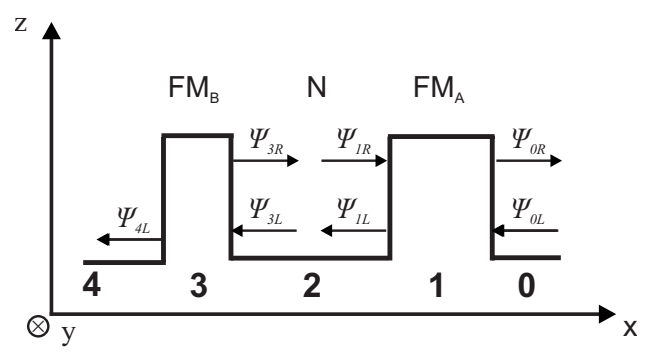

FIG. 2. Schematic diagram of the structure but with an emphasize on the amplitudes of the wave functions in each layer.

$$
\hat{r}_{A}=\left(\begin{array}{cc}
r_{u} & 0 \\
0 & r_{d}
\end{array}\right), \quad \hat{t}_{A}=\left(\begin{array}{cc}
t_{u} & 0 \\
0 & t_{d}
\end{array}\right),
$$

while for the second one, $\mathrm{FM}_{B}$,

$$
\hat{r}_{B}=\left(\begin{array}{ll}
0 & 0 \\
0 & 1
\end{array}\right), \quad \hat{t}_{B}=\left(\begin{array}{ll}
1 & 0 \\
0 & 0
\end{array}\right) .
$$

Equation (4), in fact, defines a perfect polarizer regardless of the value of the polarization $P=\left(K_{+}-K_{-}\right) /\left(K_{+}+K_{-}\right)$, where $K_{ \pm}$denote the Fermi wave vectors of the majority and/or minority spins. This is because the difference $\left|t_{u}\right|^{2}-\left|t_{d}\right|^{2}$ can be large for some optimal value of the thickness of the ferromagnet even for relatively small $P$. For the ferromagnet $A$, one should additionally rotate the matrices $\hat{r}_{A}, \hat{t}_{A}$ in spin space,

$$
\begin{aligned}
& \hat{r}_{A}(\theta)=R_{\theta} \hat{r}_{A} R_{\theta}^{-1}, \\
& \hat{t}_{A}(\theta)=R_{\theta} \hat{t}_{A} R_{\theta}^{-1},
\end{aligned}
$$

where the rotation matrix $\hat{R}_{\theta}$ is given by

$$
\hat{R}_{\theta}=\left[\begin{array}{cc}
\cos \left(\frac{\theta}{2}\right) & -\sin \left(\frac{\theta}{2}\right) \\
\sin \left(\frac{\theta}{2}\right) & \cos \left(\frac{\theta}{2}\right)
\end{array}\right] .
$$

The final expression of the matrix $Q$ defined by Eq. (2) is

$$
Q=\sqrt{z} \hat{t}_{B}\left[I_{2}-z \hat{r}_{A}(\theta) \hat{r}_{B}\right]^{-1} \hat{t}_{A}(\theta),
$$

where $z=\exp \left(i 2 \phi_{0}\right)=\exp (i \phi)$ and $I_{2}$ is the $2 \times 2$ unit matrix. It can be argued that the inverted matrix $I_{2}-z \hat{r}_{A} \hat{r}_{B}$ comes, in fact, from summing up an infinite series consisting of the amplitudes from multiple reflections, $I_{2}+\exp \left(i 2 \phi_{0}\right) \hat{r}_{A} \hat{r}_{B}$ $+\exp \left(i 4 \phi_{0}\right) \hat{r}_{A} \hat{r}_{B} \hat{r}_{A} \hat{r}_{B}+\cdots$ The explicit form of $Q$ is given by

$$
Q=\frac{\sqrt{z}}{D(z)}\left[\begin{array}{cc}
Q^{\uparrow \uparrow}(z) & Q^{\downarrow \uparrow}(z) \\
0 & 0
\end{array}\right],
$$

where

$$
\begin{aligned}
& D(z)=1-z\left(r_{d} \cos ^{2} \frac{\theta}{2}+r_{u} \sin ^{2} \frac{\theta}{2}\right), \\
& Q^{\uparrow \uparrow}(z)=t_{u} \cos ^{2} \frac{\theta}{2}+t_{d} \sin ^{2} \frac{\theta}{2}-z\left(t_{u} r_{d} \cos ^{2} \frac{\theta}{2}+r_{u} t_{d} \sin ^{2} \frac{\theta}{2}\right), \\
& Q^{\downarrow \uparrow}(z)=\frac{1}{2} \sin \theta\left[t_{u}-t_{d}+z\left(t_{d} r_{u}-t_{u} r_{d}\right)\right] .
\end{aligned}
$$

The notation $Q^{\downarrow \uparrow}$ refers to the amplitude for the transition from spin down state (minority) to spin up state (majority). The matrix $Q$ has two-nonzero elements, which results in the transmission

$$
T(z)=\frac{\left|Q^{\uparrow \uparrow}(z)\right|^{2}+\left|Q^{\downarrow \uparrow}(z)\right|^{2}}{|D(z)|^{2}} .
$$


It is understood that $T(\phi)=T\left(z=e^{i \phi}\right)$. The average transmission probability is obtained by assuming that the phase $\phi_{0}$ acquired in the spacer layer may fluctuate from 0 to $\pi$ [or $\phi \in(0,2 \pi)]$. In the ideal case, i.e., in the absence of phase averaging, there are localized levels determined by $k_{n}=n \pi / \lambda$. This is equivalent to allow $z$ to make a full unit circle in the complex plane. The expression for the average transmission is

$$
\langle T\rangle=\frac{1}{2 \pi} \int_{0}^{2 \pi} T(\phi) d \phi=\frac{1}{2 \pi i} \oint_{C} \frac{T(z)}{z} d z,
$$

and the integration is performed in the complex plane along the unit circle $|z|=1$. The function $T(z) / z$ has two poles, $z_{1}=0$ and $z_{2}=r_{d}^{*} \cos ^{2}\left(\frac{\theta}{2}\right)+r_{u}^{*} \sin ^{2}\left(\frac{\theta}{2}\right)$, inside the unit circle. In order to avoid the absolute value in the expressions, one may use the identity $z^{*}=1 / z$, since $|z|=1$, and one can apply the theorem of residuals to find the integral in Eq. (11). The final expression is

$$
\langle T\rangle=X_{1}+X_{2}+X_{3},
$$

where

$$
\begin{gathered}
X_{1}=\frac{1}{z_{2}}\left[\left|t_{u}\right|^{2} r_{d}^{*} \cos ^{2}\left(\frac{\theta}{2}\right)+\left|t_{d}\right|^{2} r_{u}^{*} \sin ^{2}\left(\frac{\theta}{2}\right)\right], \\
X_{2}=\left(t_{u}^{*}-t_{d}^{*}\right) A B_{2}, \\
X_{3}=\left[t_{u}^{*} \sin ^{2}\left(\frac{\theta}{2}\right)+t_{d}^{*} \cos ^{2}\left(\frac{\theta}{2}\right)\right] A B_{3},
\end{gathered}
$$

where

$$
\begin{gathered}
A=\left(r_{d}^{*}-r_{u}^{*}\right) \frac{\sin ^{2} \theta}{4 z_{2}\left(1-\left|z_{2}\right|^{2}\right)} \\
B_{2}=t_{u} \cos ^{2}\left(\frac{\theta}{2}\right)\left[\left|r_{d}\right|^{2} \cos ^{2}\left(\frac{\theta}{2}\right)-1\right]+t_{d} \sin ^{2}\left(\frac{\theta}{2}\right) \\
\times\left[\left|r_{u}\right|^{2} \sin ^{2}\left(\frac{\theta}{2}\right)-1\right]+\frac{1}{4}\left(t_{u} r_{u}^{*} r_{d}+t_{d} r_{d}^{*} r_{u}\right) \sin ^{2} \theta
\end{gathered}
$$

and

$$
\begin{aligned}
B_{3}= & t_{u}\left[\left|r_{d}\right|^{2} \cos ^{2}\left(\frac{\theta}{2}\right)+r_{u}^{*} r_{d} \sin ^{2}\left(\frac{\theta}{2}\right)-1\right] \\
& +t_{d}\left[1-\left|r_{u}\right|^{2} \sin ^{2}\left(\frac{\theta}{2}\right)-r_{u} r_{d}^{*} \cos ^{2}\left(\frac{\theta}{2}\right)\right] .
\end{aligned}
$$

In case when both ferromagnets are perfect polarizers, $t_{u}=1$ and $t_{d}=0\left(r_{u}=0, r_{d}=1\right)$, the transmission amplitude reduces to the well-known Slonczewski ${ }^{6}$ result, as $X_{1}=1$, while the other two terms sum up to

$$
X_{2}+X_{3}=-\frac{\sin ^{2}\left(\frac{\theta}{2}\right)}{1+\cos ^{2}\left(\frac{\theta}{2}\right)},
$$

and finally

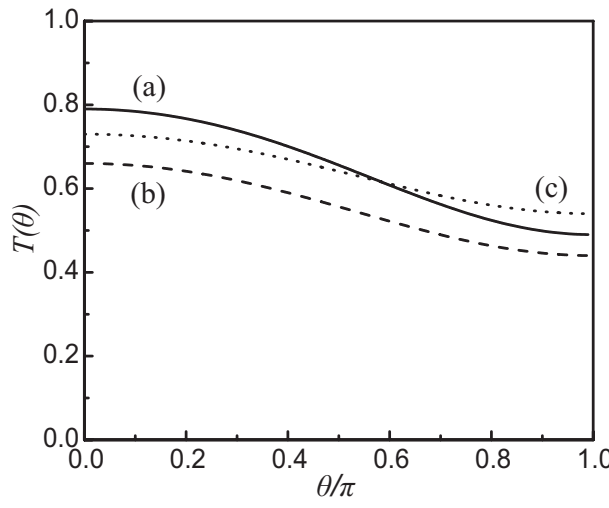

FIG. 3. Averaged transmission amplitude vs the angle $\theta$ for three different interfaces $\mathrm{Cu} / \mathrm{Co}$ : (a) (001) $\left|t_{u}\right|^{2}=0.79$ and $\left|t_{d}\right|^{2}=0.49$ (solid line); (b) (110) $\left|t_{u}\right|^{2}=0.66$ and $\left|t_{u}\right|^{2}=0.44$ (dashed line); (c) (111) $\left|t_{u}\right|^{2}=0.73$ and $\left|t_{u}\right|^{2}=0.54$ (dotted line).

$$
\langle T\rangle\left(t_{u}=1, t_{d}=0\right)=\frac{4 \cos ^{2}\left(\frac{\theta}{2}\right)}{3+\cos \theta} .
$$

Figure 3 shows the averaged transmission probability vs angle $\theta$ between the magnetizations of the two ferromagnets for three different interfaces of $\mathrm{Cu} / \mathrm{Co}$ : (001), (110), and (111). The numerical values for the minority and majority transmission probabilities are taken from Ref. 18, where they were obtained by first-principles calculations. The averaged transmission probability does not drop to zero as $\theta$ approaches $\pi$ due to the finite value of $t_{d}$ for the minority spin carrier (the values of $\left|t_{u}\right|^{2}$ and $\left|t_{d}\right|^{2}$ are given in the caption of Fig. 3).

In order to calculate the total current flowing through the structure, one should perform integration over the in-plane momentum $k_{\|}$and take into account that the transmission depends on $k_{\|}$. However, this method will not allow us to obtain analytical expressions. We will follow the approach by Slonczewski ${ }^{6}$ and assume that there are three classes of currents, $J_{a}, J_{b}$, and $J_{c}$, depending on the value of $k_{\|}$and Fermi wave vectors $K_{+}$and $K_{-}$of majority and/or minority spin subbands inside the ferromagnets. The critical wave vectors $K_{+}$and $K_{-}$depend on the exchange potentials $V_{+}$and $V_{-}$as follows:

$$
K_{ \pm}=\frac{\sqrt{2 m\left(E_{F}-V_{ \pm}\right)}}{\hbar} .
$$

Now, one can classify the currents in the following manner.

(i) Class "a": $0 \leq k_{\|}<K_{-}$. Electrons fully transmit through the double barrier structure independent of spin.

(ii) Class "b": $K_{-} \leq k_{\|}<K_{+}$. Only majority spin electrons are transmitted. flow.

(iii) Class "c": $K_{+} \leq k_{\|}$. There is no particle and/or current

The particular components of the current are therefore

$$
J_{a}=2 \frac{\hbar}{(2 \pi)^{2} m} \int_{0}^{K_{-}} \sqrt{K_{+}^{2}-k_{\|}^{2}} k_{\|} d k_{\|},
$$




$$
J_{b}=1 \frac{\hbar}{(2 \pi)^{2} m} \int_{K_{-}}^{K_{+}} \sqrt{K_{+}^{2}-k_{\|}^{2}} k_{\|} d k_{\|} .
$$

Note that $J_{b}$ is a maximal value for the case of two parallel perfect FMs. In order to obtain its contribution to the total current, it should be multiplied by the average transmission. The total electron current is given by

$$
I_{e}=e J_{a}+\langle T\rangle e J_{b} .
$$

The above procedure can be justified by the fact that the Fermi energy lies above the Stoner potentials, ${ }^{6} V_{+}, V_{-}$, and that the transmission amplitudes of the minority spin electrons sharply decrease when the in-plane momentum $k_{\|}$ crosses the value $K_{-}$. The ratio $J_{a} / J_{b}$ can be estimated from the polarization $P$,

$$
\frac{J_{a}}{J_{b}}=\frac{2 K_{+}^{3}}{\left(K_{+}^{2}-K_{-}^{2}\right)^{3 / 2}}-2=\frac{(1+P)^{3}}{4 P^{3 / 2}}-2 .
$$

\section{SPIN TORQUE}

Finally, we arrive at the spin torque acting on the ferromagnets. Such as in Ref. 11, the thickness of the ferromagnet $A$ is taken to be larger than that of ferromagnet $B$, so that the intralayer exchange and anisotropy forces will keep $\hat{\mathbf{m}}_{A}$ fixed. Hence, one is interested only in the torque acting on ferromagnet $B$, which is equal to the difference in the spin fluxes on both sides of the ferromagnet, ${ }^{11}$

$$
\frac{\partial \vec{\tau}_{B}}{\partial V_{b}}=\vec{J}_{2 s}-\vec{J}_{4 s}
$$

for small applied bias $V_{b}$. Here, the positive value of the torque is adopted for particles flowing along the positive axis (from left to right). Note that the Landauer formula was used to obtain Eqs. (22a) and (22b), which is valid for small $V_{b}$. Then, $J_{2,4 s}$ have dimensions of conductance and this is the reason that instead of $\tau_{B}$, its differential appears in Eq. (25). In the case considered here, $\mathrm{FM}_{B}$ is a perfect polarizer and $J_{4 s}=0$ while the spin polarization in region 2 is found if the "transfer matrix" $\Omega$, which connects regions 2 and lead 0 , is known. Its general form is

$$
\Omega=\sqrt{z}\left[I_{2}-z \hat{r}_{A}(\theta) \hat{r}_{B}\right]^{-1} \hat{t}_{A}(\theta)
$$

using the adopted notations. It has the same denominator as the matrix $Q$,

$$
\Omega=\frac{\sqrt{z}}{D(z)}\left(\begin{array}{ll}
\Omega^{\uparrow \uparrow} & \Omega^{\downarrow \uparrow} \\
\Omega^{\uparrow \downarrow} & \Omega^{\downarrow \downarrow}
\end{array}\right),
$$

with the first row being identical with that of $Q$, i.e., $Q^{\uparrow \uparrow}=\Omega^{\uparrow \uparrow}$ and $Q^{\downarrow \uparrow}=\Omega^{\downarrow \uparrow}$, while the other two matrix elements are given by

$$
\begin{gathered}
\Omega^{\uparrow \downarrow}=\frac{1}{2} \sin \theta\left(t_{u}-t_{d}\right), \\
\Omega^{\downarrow \downarrow}=t_{d} \cos ^{2} \frac{\theta}{2}+t_{u} \sin ^{2} \frac{\theta}{2} .
\end{gathered}
$$

To evaluate the spin torque, one should find the expectation value of $\vec{s}$, i.e.,

$$
T_{s}(z)=\operatorname{Tr}\left(\hat{s} \Omega \Omega^{\dagger}\right) .
$$

Only the $x$ component of the spin torque is nonzero, which leads to

$$
T_{s}(z)=\frac{\operatorname{Re}\left\{\Omega^{\uparrow \uparrow} \Omega^{* \uparrow \downarrow}+\Omega^{\downarrow \uparrow} \Omega^{* \downarrow \downarrow}\right\}}{|D(z)|^{2}}
$$

and performs the same averaging procedure to find $\left\langle T_{s}\right\rangle$, as in previous case. The spin density current is composed of only b class currents, which is $J_{2 s}=\left\langle T_{s}\right\rangle J_{b}$ and

$$
J_{2 s}=\frac{\sin \theta J_{b}}{2\left(1-\left|z_{2}\right|^{2}\right)}\left[Y_{1}-\left(t_{u}^{*}-t_{d}^{*}\right) B_{2}\right],
$$

with $B_{2}$ defined in Eq. (17) while

$$
Y_{1}=\left[t_{d}^{*} \cos ^{2}\left(\frac{\theta}{2}\right)+t_{u}^{*} \sin ^{2}\left(\frac{\theta}{2}\right)\right]\left[\left(t_{d} r_{u}-t_{u} r_{d}\right) z_{2}+t_{u}-t_{d}\right] .
$$

Recall that $z_{2}$ is the second zero of the denominator $|D(z)|^{2}$ and was defined earlier. At this point, it is useful to introduce a scalar function $g$ as proposed by Slonczewski ${ }^{6}$ as a spin-angular momentum acting on a ferromagnet per total current $\left(I_{e}\right)$ and taking into account the relative orientation of the ferromagnets,

$$
\frac{\partial \vec{\tau}_{B}}{\partial V_{b}}=g(\theta) \frac{I_{e}}{e} \hat{\mathbf{m}}_{B} \times\left(\hat{\mathbf{m}}_{A} \times \hat{\mathbf{m}}_{B}\right) .
$$

Here, $\hat{\mathbf{m}}_{A, B}$ are unit vectors of magnetization. Since the magnetization of both ferromagnets are in plane, the expression for $g(\theta)$ becomes

$$
g(\theta)=\frac{1}{\sin \theta} \frac{\left\langle T_{s}\right\rangle}{J_{a} / J_{b}+\langle T\rangle} .
$$

In the limit $t_{u} \rightarrow 1, t_{d} \rightarrow 0$, this expression reduces to the result of Slonczewski, ${ }^{6}$

$$
g(\theta)=\left[-4+(1+P)^{3}\left(3+\hat{\mathbf{m}}_{A} \cdot \hat{\mathbf{m}}_{B}\right) /\left(4 P^{3 / 2}\right)\right]^{-1},
$$

since the average spin transmission (along the $x$ axis) takes the simpler form,

$$
\left\langle T_{s}\right\rangle=\frac{\sin \theta}{3+\cos \theta} .
$$

Often, authors consider only the torque per unit of current $I_{e}$, in fact, $g$ but without the sine factor in Eq. (35),

$$
\tau_{B}^{\prime}(\theta)=\frac{e}{I_{e}} \frac{\partial \tau_{B}^{x}}{\partial V_{b}},
$$

which is measured in units of $\hbar /(2 e)$. In a very special and ideal case when both ferromagnets are perfect polarizers and $P=1$, this factor is equal to $\tan (\theta) / 2$ and has a singularity at $\theta=\pi$ as previously noted. ${ }^{6,11}$

In Fig. 4, we present the spin torque per unit current as a function of angle $\theta$ for three cases: (a) ideal $\tan (\theta) / 2$ case, 


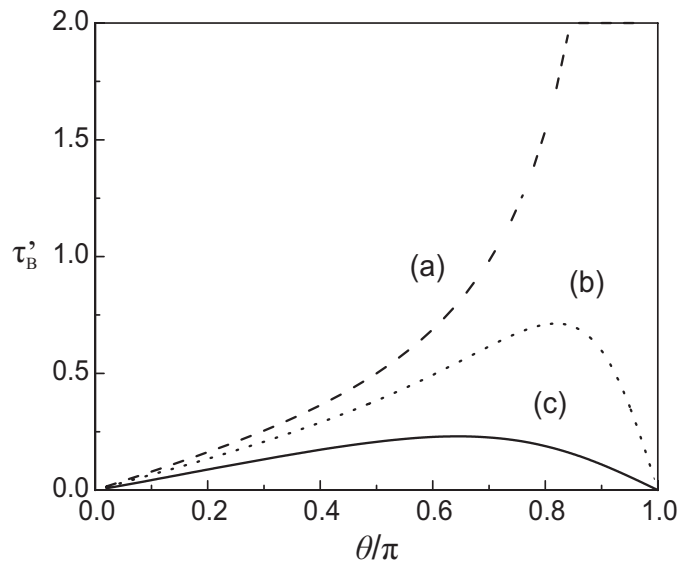

FIG. 4. Spin-torque per unit current vs the angle $\theta$ between the magnetization of $\mathrm{FM}_{A}$ and $\mathrm{FM}_{B}$ for three cases: (a) both FMs are perfect polarizers and polarization $P=1$; (b) both FMs are perfect polarizers but $P=0.6$; and (c) asymmetric case: $\left|t_{u}\right|^{2}\left(\mathrm{FM}_{A}\right)=0.3$, $\left|t_{d}\right|^{2}\left(\mathrm{FM}_{A}\right)=0.01$, and polarization $P=0.6$.

(b) case when both FMs are perfect polarizers but with finite $P=0.6$, and (c) $\mathrm{FM}_{A}$ has the following parameters $\left|t_{u}\right|^{2}=0.3$ and $\left|t_{d}\right|^{2}=0.01$, such as in Ref. 11. Note, at this point, that there is no direct relation between $t_{u, d}$ and the polarization $P$, since the former also depends on the thickness of the ferromagnet. It is obvious that in the present asymmetric case, the spin torque is smaller for the same polarization $P$. The values of $g(\theta=0, \pi)$ are important to assess the critical current density $J_{\mathrm{cr}}$ needed for magnetization reversal of the free layer, as $J_{\mathrm{cr}} \propto 1 / g(0, \pi)$. In Table I, we give the spin-torque $g$ factor for three different $\mathrm{Cu} / \mathrm{Co}$ interfaces: (001), (110), and (111) at two characteristic angles, parallel $(\theta=0)$, and antiparallel $(\theta=\pi)$. The values are calculated for transmission probabilities given in Ref. 18 (see the caption of Fig. 3) and for polarization ${ }^{19}$ of cobalt at $P=0.35$. For comparison, we give $g_{0}$ for the symmetric case when both ferromagnets have complete transmission of majority spins, which is independent of the type of interface.

Seki et al. ${ }^{20}$ observed and characterized magnetization switching in $\mathrm{FePt} / \mathrm{Au} / \mathrm{FePt}$ nanopillars. They found relatively good agreement with the Slonczewski ${ }^{6}$ formula for the experimental values: theory: $J_{\mathrm{cr}}^{\mathrm{P}-\mathrm{AP}}=-2.0 \times 10^{8} \mathrm{~A} / \mathrm{cm}^{2}$ and $\quad J_{\text {cr }}^{\mathrm{AP}-\mathrm{P}}=0.4 \times 10^{8} \mathrm{~A} / \mathrm{cm}^{2} ; \quad$ experiment: $J_{\mathrm{cr}}^{\mathrm{P}-\mathrm{AP}}$ $=0.9 \times 10^{8} \mathrm{~A} / \mathrm{cm}^{2}$ and $J_{\mathrm{cr}}^{\mathrm{AP}-\mathrm{P}}=1.7 \times 10^{8} \mathrm{~A} / \mathrm{cm}^{2}$, with the exception that in the experiment, the critical current for switching from parallel to antiparallel alignment is smaller than in

TABLE I. Spin-torque $g$ factor corresponding to various $\mathrm{Cu} / \mathrm{Co}$ interfaces and the $\mathrm{Au} / \mathrm{Fe}(001)$ interface for $\theta=0, \pi$. The values of $g_{0}$ is also given for two perfect ferromagnets. In all cases, polarization is $P=0.35$.

\begin{tabular}{llll}
\hline \hline Interface & $g(0)$ & $g(\pi)$ & $g_{0}(0) / g_{0}(\pi)$ \\
\hline $\mathrm{Cu} / \mathrm{Co}(001)$ & 0.033 & -0.063 & $0.13 /-0.51$ \\
$\mathrm{Cu} / \mathrm{Co}(110)$ & 0.03 & -0.044 & $0.13 /-0.51$ \\
$\mathrm{Cu} / \mathrm{Co}(111)$ & 0.024 & -0.036 & $0.13 /-0.51$ \\
$\mathrm{Au} / \mathrm{Fe}(001)$ & 0.056 & -0.2 & $0.13 /-0.51$ \\
\hline \hline
\end{tabular}

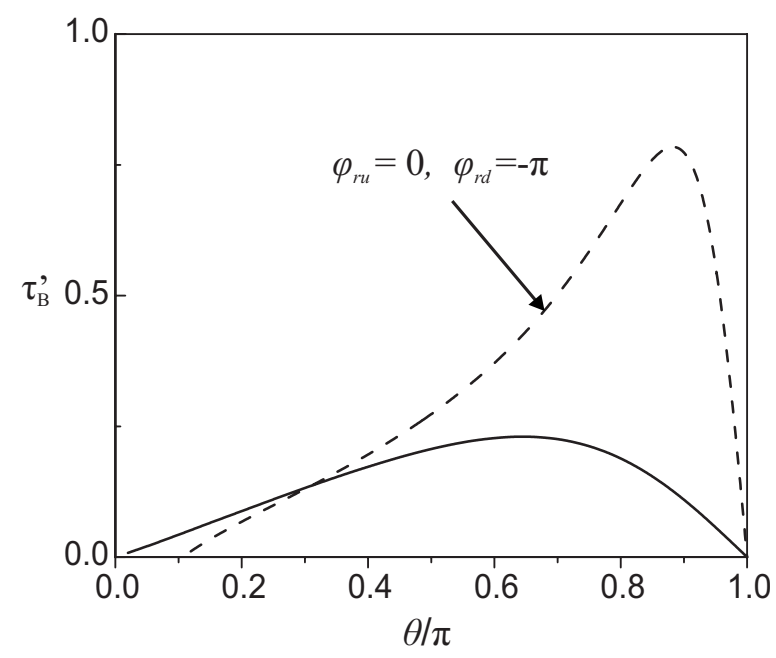

FIG. 5. The influence of the phases of $r_{u}$ and $r_{d}$ on the dependence of the spin torque vs angle $\theta$ : (a) $\varphi_{r u}=0, \varphi_{r d}=0, P=0.6$, $\left|t_{u}\right|^{2}=0.3$, and $\left|t_{d}\right|^{2}=0.01$; (b) $\varphi_{r u}=0$ and $\varphi_{r d}=-\pi$, other parameters being the same as in case (a).

the opposite direction. However, one of the pillars was significantly longer $(20 \mathrm{~nm})$ than the other one $(1.5 \mathrm{~nm})$ in the experimental setup and one may expect that the first ferromagnet may have a smaller transmission probability. For instance, if we take $\left|t_{u}\right|^{2}=0.84$ and $\left|t_{d}\right|^{2}=0.17$ for the $\mathrm{Au} / \mathrm{Fe}$ interface, the ratio $g(\pi) / g(0)=-4.0$ takes a somewhat smaller value (in absolute sense) than in the symmetric ideal case $g_{0}(\pi) / g_{0}(0)=-4$.8. However, the exact probability for the particular case of the $\mathrm{Au} / \mathrm{PtFe}$ interface is not known. Unfortunately, in this case too, we find that the critical current needed to switch from parallel to antiparallel alignment is larger than that from antiparallel to parallel contrary to the experimental data.

In the calculations, up to now, the phases of $t_{u}, t_{d}, r_{u}$, and $r_{d}$ are neglected, i.e., they are assumed to be zero. Even though the averaging procedure is performed for the phase acquired during passage in the normal layer, the electron still sees the whole structure quantum mechanically. The phases of the transmission amplitudes do not influence the final results like in the spin-independent case. This is because the transmission matrix $\hat{t}_{A}$ is diagonal and it does not appear in the denominator of Eq. (7), so that the phases are canceled out in $\langle T\rangle$ due to the absolute values or in $\left\langle T_{s}\right\rangle$ because of conjugations. Furthermore, if phases of reflection for both spin up and spin down have the same value $\varphi_{r u}=\varphi_{r d}$, they are easily absorbed in the variable $z$ so that it does not have any influence on the spin torque either. Only if the phases $\varphi_{r u}$ and $\varphi_{r d}$ have a different sign [within the range $(-\pi, \pi)$ ], it may enhance the spin torque or the $g$ factor. We find that maximum improvement is achieved for $\varphi_{r u}=0$ and $\varphi_{r d}=-\pi$, as shown in Fig. 5.

\section{STRUCTURES WITH A PERPENDICULAR POLARIZER}

Recently, spin-valve structures containing a perpendicular polarizer made of $\mathrm{FePt}$ (Refs. 21 and 22) or $\mathrm{Pt} /(\mathrm{Co} / \mathrm{Pt})_{5}$ 


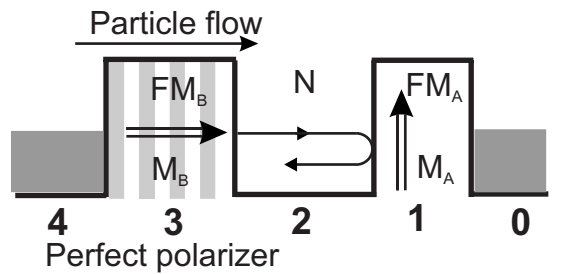

FIG. 6. Schematic diagram of the spin-valve structure containing a perpendicular polarizer.

multilayers ${ }^{23}$ have been realized. These structures have three basic elements: the perpendicular polarizer, a free layer, and an analyzer layer whose magnetization is pinned. The last two ferromagnetic layers have magnetization in plane and are thinner than the polarizer. The fixed layer is usually designed $^{23}$ to have negligible effect on the total spin-torque effect by reducing its length well below the spin diffusion length. Thus, one may consider only polarizers and the free layer when evaluating the relevant physical quantities.

Since the contribution of the fixed layer to the total spintorque effect is usually neglected, ${ }^{23}$ one may consider only the polarizer and the free layer when evaluating relevant physical quantities. In contrast to previous case, in Sec. III, the spin torque does not depend on the orientation of the magnetization of the free layer $\left(\hat{\mathbf{m}}_{A}\right)$ as long as it is in plane and it is taken to be aligned along the $z$ axis. Another important difference is that one is interested in the spin torque acting on the thinner free layer, i.e., ferromagnet $A$. The matrix that connects the external leads is

$$
Q_{\perp}=\frac{\sqrt{z}}{2-z\left(r_{u}+r_{d}\right)}\left[\begin{array}{ll}
t_{u}\left(1-z r_{d}\right) & t_{u}\left(z r_{d}-1\right) \\
t_{d}\left(z r_{u}-1\right) & t_{d}\left(1-z r_{u}\right)
\end{array}\right] .
$$

The expression for the average transmission is the same as in previous case when one inserts for the angle $\theta$ the value $\theta=\pi / 2$ in Eqs. (13)-(18). The spin torque is nonzero only along the $x$ axis,

$$
\frac{\partial \tau_{B}^{x}}{\partial V_{b}}=\left(U_{1}+U_{2}\right)\left(1-\operatorname{Re}\left\{t_{u} \cdot t_{d}^{*}\right\}\right)
$$

where

$$
U_{1}=-\frac{r_{u}^{*}}{r_{u}^{*}+r_{d}^{*}}
$$

and

$$
U_{2}=\frac{\left(r_{u}^{*}-r_{d}^{*}\right)\left(2-\left|r_{d}\right|^{2}-r_{u}^{*} r_{d}\right)}{\left(r_{u}^{*}+r_{d}^{*}\right)\left(4-\left|r_{u}+r_{d}\right|^{2}\right)} .
$$

When the particle flow is in the opposite direction, i.e., from the right to left in Fig. 6, the spin torque assumes the following form:

$$
\frac{\partial \tau_{B}^{x \leftarrow}}{\partial V_{b}}=\left(V_{1}+V_{2}\right)
$$

where
TABLE II. Spin-torque $g_{\perp}, g_{\perp}^{\leftarrow}$ factor corresponding to various $\mathrm{Cu} / \mathrm{Co}$ interfaces for structure containing a perpendicular polarizer and for two directions of electron flow. For comparison, the value of $g(\pi / 2)$ is given using the formula for the in-plane polarizer. In all cases, polarization is $P=0.35$.

\begin{tabular}{llll}
\hline \hline Interface & $g_{\perp}$ & $g_{\perp}^{\leftarrow}$ & $g(\pi / 2)$ \\
\hline $\mathrm{Cu} / \mathrm{Co}(001)$ & -0.11 & -0.098 & 0.047 \\
$\mathrm{Cu} / \mathrm{Co}(110)$ & -0.15 & -0.14 & 0.036 \\
$\mathrm{Cu} / \mathrm{Co}(111)$ & -0.11 & -0.10 & 0.030 \\
$\mathrm{Au} / \mathrm{Fe}(001)$ & -0.18 & -0.11 & 0.12 \\
\hline \hline
\end{tabular}

$$
V_{1}=\frac{\left|t_{u}\right|^{2} r_{d}^{*}}{r_{u}^{*}+r_{d}^{*}}
$$

and

$$
\begin{aligned}
V_{2}= & -\frac{r_{d}^{*}}{r_{u}^{*}+r_{d}^{*}} \frac{1}{4-\left|r_{u}+r_{d}\right|^{2}}\left\{\left|t_{u}\right|^{2}\left(4-\left|r_{d}\right|^{2}-r_{u}^{*} r_{d}\right)\right. \\
& \left.+\left|t_{d}\right|^{2} r_{u}\left(r_{u}^{*}+r_{d}^{*}\right)\right\} .
\end{aligned}
$$

All of the presented analytical expressions were numerically checked (including those from Sec. III). In Table II, we present values of $g_{\perp}$ and $g_{\perp}^{\leftarrow}$ for two different material combinations, $\mathrm{Cu} / \mathrm{Co}$ and $\mathrm{Au} / \mathrm{Fe}$, and various interfaces. In addition, the value of $g(\theta=\pi / 2)$ from Sec. III is also given, which corresponds to the arrangement when the magnetization of the polarizer is aligned along the $y$ axis. The values of $g(\theta=\pi / 2)$, in most cases, underestimate the actual value of $g_{\perp}\left(\right.$ or $\left.g_{\perp}^{\leftarrow}\right)$. This can be explained, in part, in the following manner: just consider the usual case when the magnetization of the two FMs are pointing along the $z$ axis, such as in Sec. III, and assume there are no spin-flip processes. If there had not been for multiple reflections inside the spacer, it would have not been possible to transfer a torque from one ferromagnet to the other since the quantity $s_{z}$ is conserved. Through phase averaging and multiple passes of the electron in the normal layer, we have shown that it is possible to transfer a torque along the $x$ axis. However, if there is already a polarization along the current flow, one may expect that spin torque is enhanced. Of course, it depends also on the particular values of the transmission amplitudes of the majority and the minority spins, $t_{u}$ and $t_{d}$. Indeed, Seki et $a l .{ }^{22}$ observed a decrease of the critical current when the perpendicular polarizer is introduced in the structure as they had prepared two samples, one with and the other without a $L 1_{0}$-FePt polarizing layer.

\section{SUMMARY AND CONCLUSIONS}

In conclusion, we studied the spin torque in FM/N/FM structures within the ballistic regime under the assumption that spin-flip and mean-free path lengths are larger than the normal spacer length $\left(D_{N}\right)$ and in the absence of scattering centers. For example, this is satisfied in sandwich structures where copper is used as a normal layer since $l_{\mathrm{sf}} \approx 350$ at room temperature ${ }^{24}$ and $l_{\mathrm{sf}} \approx 450 \mathrm{~nm}$ at $T=4.2 \mathrm{~K},{ }^{25}$ while 
$l_{\mathrm{mf}} \approx 110 \mathrm{~nm}(T=4.2 \mathrm{~K}$; Ref. 24). Analytical formulas for total and spin current are derived for the case when one of the ferromagnets is a full polarizer. It is shown that the phases of the reflection coefficients must be taken into account. The values obtained under the mentioned assumptions give more stringent conditions for the critical current $J_{\text {cr }}$ needed for magnetization reversal. In addition, we extended the model for multilayers having a polarizer whose magnetization is along the current flow, which was recently realized. We show that in most practical cases of interest, the presence of a perpendicular polarizer has a favorable effect on the $g$ factor and on the lowering of the critical current. In all presented situations, the analytical values may serve as theoretical upper bounds for the spin torque in realistic structures due to the approximation of no scattering centers.

\section{ACKNOWLEDGMENTS}

One of the authors (M.K.) thanks W. Van Roy and L. Lagae for the fruitful discussions. This work is supported by the Flemish Science Foundation (FWO-Vl) and the Interuniversity Attraction Poles Program (IAP)-Belgian StateBelgian Science Policy. *predrag.krstajic@ua.ac.be

†rancois.peeters@ua.ac.be

${ }^{1}$ I. Žutić, J. Fabian, and S. D. Sarma, Rev. Mod. Phys. 76, 323 (2004).

${ }^{2}$ A. E. Berkowitz, J. R. Mitchell, M. J. Carey, A. P. Young, D. Rao, A. Starr, S. Zhang, F. E. Spada, F. T. Parker, A. Hutten, and G. Thomas, J. Appl. Phys. 73, 5320 (1993).

${ }^{3}$ M. N. Baibich, J. M. Broto, A. Fert, F. Nguyen Van Dau, F. Petroff, P. Eitenne, G. Creuzet, A. Friederich, and J. Chazelas, Phys. Rev. Lett. 61, 2472 (1988).

${ }^{4}$ G. Binasch, P. Grünberg, F. Saurenbach, and W. Zinn, Phys. Rev. B 39, 4828 (1989).

${ }^{5}$ L. Berger, Phys. Rev. B 54, 9353 (1996).

${ }^{6}$ J. C. Slonczewski, J. Magn. Magn. Mater. 159, L1 (1996).

${ }^{7}$ J. A. Katine, F. J. Albert, R. A. Buhrman, E. B. Myers, and D. C. Ralph, Phys. Rev. Lett. 84, 3149 (2000).

${ }^{8}$ E. B. Myers, D. C. Ralph, J. A. Katine, R. N. Louie, and R. A. Buhrman, Science 285, 867 (1999).

${ }^{9}$ M. AlHajDarwish, H. Kurt, S. Urazhdin, A. Fert, R. Loloee, W. P. Pratt, Jr., and J. Bass, Phys. Rev. Lett. 93, 157203 (2004).

${ }^{10}$ D. Chiba, Y. Sato, T. Kita, F. Matsukura, and H. Ohno, Phys. Rev. Lett. 93, 216602 (2004).

${ }^{11}$ X. Waintal, E. B. Myers, P. W. Brouwer, and D. C. Ralph, Phys. Rev. B 62, 12317 (2000).

12 J. C. Slonczewski, J. Magn. Magn. Mater. 247, 324 (2002).

${ }^{13}$ J. Xiao, A. Zangwill, and M. D. Stiles, Phys. Rev. B 70, 172405
(2004).

${ }^{14}$ A. Brataas, Y. V. Nazarov, and G. E. W. Bauer, Eur. Phys. J. B 22, 99 (2001)

${ }^{15}$ J. Barnaś, A. Fert, M. Gmitra, I. Weymann, and V. K. Dugaev, Phys. Rev. B 72, 024426 (2005).

${ }^{16}$ D. Waldron, P. Haney, B. Larade, A. MacDonald, and H. Guo, Phys. Rev. Lett. 96, 166804 (2006).

${ }^{17}$ P. M. Haney, D. Waldron, R. A. Duine, A. S. Núñez, H. Guo, and A. H. MacDonald, Phys. Rev. B 76, 024404 (2007).

${ }^{18}$ M. D. Stiles, J. Appl. Phys. 79, 5805 (1996).

${ }^{19}$ P. M. Tedrow and R. Meservey, Phys. Rev. B 7, 318 (1973).

${ }^{20}$ T. Seki, S. Mitani, K. Yakushiji, and K. Takanashi, J. Appl. Phys. 99, 08G521 (2006).

${ }^{21}$ T. Seki, S. Mitani, K. Yakushiji, and K. Takanashi, Appl. Phys. Lett. 88, 172504 (2006).

${ }^{22}$ T. Seki, S. Mitani, K. Yakushiji, and K. Takanashi, Appl. Phys. Lett. 89, 172504 (2006).

${ }^{23}$ D. Houssameddine, U. Ebels, B. Delaët, B. Rodmacq, I. Firastrau, F. Ponthenier, M. Brunet, C. Thirion, J.-P. Michel, L. Prejbeanu-Buda, M.-C. Cyrille, O. Redon, and B. Dieny, Nat. Mater. 6, 441 (2007).

${ }^{24}$ F. J. Jedema, M. S. Nijboer, A. T. Filip, and B. J. van Wees, Phys. Rev. B 67, 085319 (2003).

${ }^{25}$ Q. Yang, P. Holody, S.-F. Lee, L. L. Henry, R. Loloee, P. A. Schroeder, W. P. Pratt, and J. Bass, Phys. Rev. Lett. 72, 3274 (1994). 